\title{
Plântulas de espécies arbóreas na floresta ciliar do rio Mogi Guaçu, Pirassununga, SP, Brasil ${ }^{1}$
}

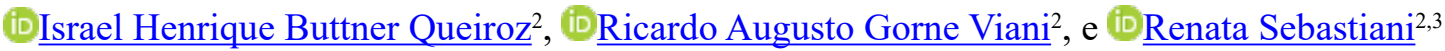

Como citar: Queiroz, I.H.B., Viani, R.A.G., \& Sebastiani, R. 2021. Plântulas de espécies arbóreas na floresta ciliar do rio Mogi Guaçu, Pirassununga, SP, Brasil. Hoehnea 48: e1122020. https://doi.org/10.1590/2236-8906-112/2020

\begin{abstract}
Plântulas de espécies arbóreas na floresta ciliar do rio Mogi Guaçu, Pirassununga, SP, Brasil). A permanência de uma espécie vegetal, em uma comunidade florestal, depende do seu número de indivíduos e de sua distribuição nos vários tamanhos de indivíduos, inclusive plântulas. Plântulas e sementes de um remanescente florestal em Pirassununga foram coletadas e analisadas quanto ao grau de ameaça, altura possível do indivíduo adulto, ocorrência em fitofisionomias e região e aspectos morfológicos. Foram coletadas 116 plântulas resultando em 39 morfoespécies, e a germinação de sementes coletadas proporcionou 40 espécies. O presente estudo trouxe novidades quanto aos aspectos morfológicos, à identificação e à distribuição de plântulas de espécies arbóreas em área de floresta ciliar na Bacia do Rio Mogi Guaçu e no Estado de São Paulo. Keywords: biodiversidade, conservação, estrato arbóreo, regenerante
\end{abstract}

RESUMO - (Seedlings of tree species in the riparian forest of the Mogi Guaçu river, Pirassununga, São Paulo State, Brasil). The permanence of a plant species in a forest community depends on its number of specimens and its distribution in the various sizes of individuals, including seedlings. Seedlings and seeds from a forest remnant in Pirassununga were collected and analyzed for the degree of threat, possible height of the adult individual, occurrence in phytophysiognomies and region and morphological aspects. 116 seedlings available in 39 morphospecies were collected, and the germination of collected seeds provided 40 species. The present study brought news regarding the morphological aspects, the identification and the distribution of seedlings of tree species in a riparian forest area in the Mogi Guaçu River Basin and in the State of São Paulo. Palavras-chave: arboreal layer, biodiversity, conservation, regenerating

\section{Introdução}

No Estado de São Paulo, há cerca de $13 \%$ da superfície com vegetação original altamente fragmentada, representada por 85.290 fragmentos com menos de 20 hectares (ha) e apenas 539 fragmentos com mais de 500 ha (Nalon et al. 2008). Quanto à relação da vegetação com os recursos hídricos, 14 das 22 bacias hidrográficas do Estado possuem menos de $10 \%$ de sua superfície com remanescentes, restando apenas duas com mais de 50\% de cobertura vegetal (Nalon et al. 2008). A supressão da vegetação natural alcançou também as florestas ciliares, gerando preocupação por causa do aumento do processo erosivo, diminuição da fertilidade dos solos e assoreamento dos cursos d'água (Guidotti et al. 2020).

A floresta ciliar ocorre nas margens de rios e nascentes, geralmente é constituída por plantas de casca lisa e tronco cônico, e apresenta muitas palmeiras, trepadeiras e epífitas (IBGE 2012). A floresta ciliar atua na estabilidade dos solos marginais e das ribanceiras, prevenindo a erosão e o assoreamento; na regulação da quantidade de nutrientes, sedimentos e poluentes que são transportados para os cursos d'água; interceptação e absorção da radiação solar, contribuindo para a estabilização térmica dos cursos d'água; no sustento e desenvolvimento dos organismos aquáticos e da fauna; e na melhora da infiltração da água no solo (Cardoso-Leite et al. 2004, Nóbrega et al. 2007).
A alta diversidade das florestas tropicais é consequência das espécies raras, as quais possuem pouca abundância e ou distribuição geográfica restrita (Higuchi 2003, Ter Steege et al. 2013). A permanência de uma espécie vegetal, em uma comunidade florestal, depende do número de indivíduos e de sua distribuição nos vários tamanhos, ou seja, desde plântulas até indivíduos adultos. Nas florestas tropicais, fatores como luminosidade, padrão de produção e dispersão de sementes, predadores de sementes e de plântulas, danos físicos, histórico de perturbação e idade da floresta secundária influenciam na abundância e riqueza de plântulas das espécies arbóreas (Alves \& Metzger 2006).

O banco de plântulas geralmente compreende as espécies não pioneiras ou clímax que germinam sob o dossel e podem se estabelecer e permanecer sob a sombra da floresta durante anos (Viani \& Rodrigues 2009). Mas, no caso de formações altamente fragmentadas, com histórico de degradação ou com terreno acentuadamente em declive, há espécies de outros estágios da sucessão florestal na comunidade de plântulas. Há outro grupo de espécies, as pioneiras, cujas sementes dependem de ambientes abertos como as clareiras para germinarem, sendo intolerantes à sombra e em geral ausentes sob o dossel florestal (Viani \& Rodrigues 2009).

1 Trabalho de Conclusão de Curso do primeiro Autor

2 Universidade Federal de São Carlos, Centro de Ciências Agrárias, Rodovia Anhanguera, Km 174, 13600-970 Araras, SP, Brasil

3 Autora para correspondência: sebastiani@ufscar.br 
Trabalhos com plântulas e indivíduos juvenis de espécies arbóreas nas regiões sul e sudeste do Brasil já foram realizados com diferentes objetivos (Silva Junior 2002, Araujo et al. 2004, Alves \& Metzger 2006, Higuchi et al. 2006, Salles \& Schiavini 2007, Martins et al. 2008, Viani \& Rodrigues 2009, Garcia et al. 2011, Leyser et al. 2012, Santos et al. 2012). No entanto, são escassos os estudos detalhados sobre aspectos morfológicos das plântulas de espécies arbóreas nativas, tais como Frigieri et al. (2016). Apesar da alta diversidade biológica, são poucos os trabalhos que forneçam informações sobre as espécies nativas em seus estágios iniciais, os quais são fundamentais para outras pesquisas como a taxonomia, ecologia e aspectos práticos, como a identificação de plântulas em viveiros (Frigieri et al. 2016).

Considerando o cenário de degradação e fragmentação da vegetação nativa no Estado de São Paulo, assim como a importância da floresta ciliar e a eminente perda de sua biodiversidade, o objetivo do presente estudo foi realizar um levantamento de plântulas de espécies arbóreas em uma área de floresta ciliar do Rio Mogi Guaçu no município de Pirassununga, a fim de ampliar o conhecimento sobre a biodiversidade regional, colaborar com informações sobre a estratificação da floresta ao longo da sucessão florestal e trazer aspectos morfológicos importantes para identificação das espécies de plântulas.

\section{Material e métodos}

O município de Pirassununga localiza-se na região nordeste do Estado de São Paulo, apresenta clima temperado mesotérmico, moderadamente chuvoso e com inverno seco (Batalha et al. 1997) e está inserido na Unidade de Gerenciamento de Recursos Hídricos (UGRHI) do Rio Mogi-Guaçu, contendo apenas 6,2\% (cerca 4.500 ha) de sua superfície cobertos por vegetação nativa (Kronka et al. 2005).

A Guarnição da Aeronáutica de Pirassununga está inserida na UGRHI do Rio Mogi Guaçu e é importante para a conservação da biodiversidade, pois está em uma região de ecótono com fragmentos de cerrado e floresta estacional semidecidual e a grande extensão de floresta ciliar contínua que interliga esses fragmentos florestais é imprescindível para a manutenção da biodiversidade local. A Guarnição da Aeronáutica de Pirassununga possui uma área total de 6.500 ha, dos quais 2.253 ha constituem fragmentos de vegetação natural (E. Fernandes, dados não publicados). Há cerca de 1.554 ha compreendendo floresta estacional semidecidual, cerrado, floresta ciliar e transições entre essas fitofisionomias. O presente estudo foi conduzido na floresta ciliar que é contínua a um fragmento de floresta estacional semidecidual com cerca de 140 ha (figura 1).
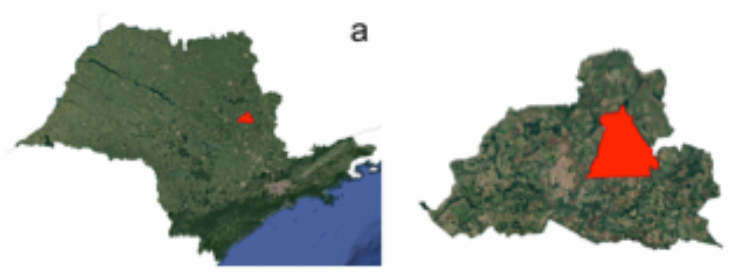

b
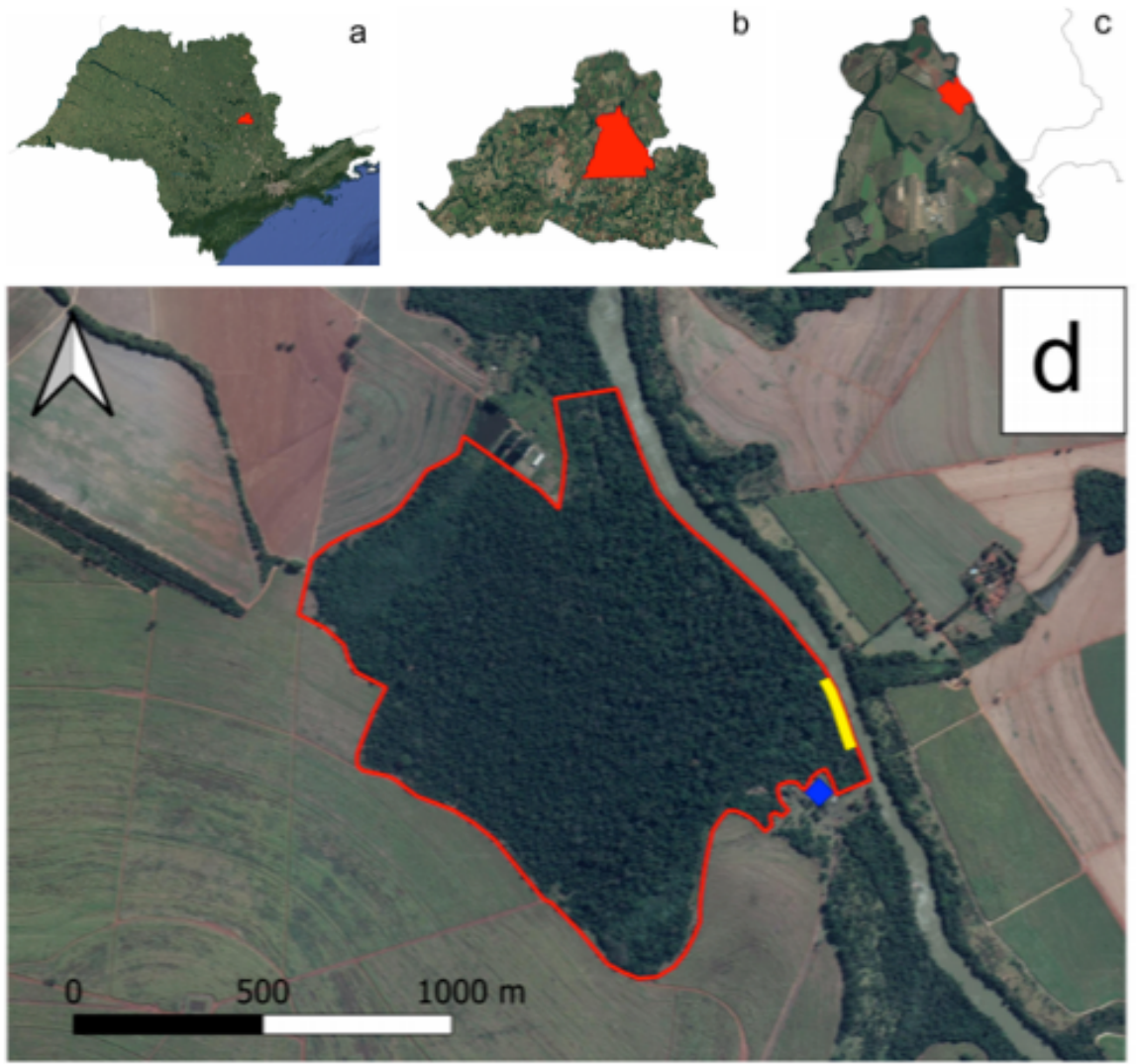

Figura 1. a: Estado de São Paulo, Brasil, com a região de Pirassununga destacada em vermelho. b: Pirassununga com a Guarnição da Aeronáutica destacada em vermelho. c: Guarnição da Aeronáutica com o Fragmento de Floresta Estacional Semidecidual (FES) contínuo à Floresta Ciliar (FC) destacado em vermelho. d: Fragmento de FES contínuo à FC delimitado em vermelho com a área de estudo (retângulo amarelo), Estação de Tratamento de Água (losango azul) e Rio Mogi Guaçu (seta azul).

Figure 1. a: State of São Paulo, Brazil, with Pirassununga highlighted in red. b: Pirassununga with the Guarnição da Aeronáutica de Pirassununga in red. c: Guarnição da Aeronáutica de Pirassununga with the Seasonal Semideciduous Forest (FES) fragment continuous to the Riparian Forest (FC) in red. d: FES Fragment continuous to the FC delimited in red and the study area (yellow rectangle), the Water Treatment Station (blue diamond) and Mogi Guaçu River (blue arrow). 
Nessa área a vegetação do sub-bosque é densa, impossibilitando a nítida visualização do dossel e do estrato emergente, além da maioria dos indivíduos arbóreos adultos serem muito altos, inviabilizando o acesso a sua copa sem que seja por meio de escalada.

A área estudada foi uma parcela de aproximadamente 200 x 30 m (metros) ao longo do curso d'água, a $100 \mathrm{~m}$ de distância da Estação de Tratamento de Água da Guarnição, paralela ao Rio Mogi Guaçu, estando há $5 \mathrm{~m}$ de distância deste (figura 1). Assim como adotou Viani \& Rodrigues (2009), definiu-se como plântula os indivíduos com altura entre 5 e $30 \mathrm{~cm}$ (centímetros) a partir do nível do solo até a última gema à amostragem. Entre os meses de janeiro e agosto de 2018 foram realizadas visitas mensalmente na área de estudo. A seleção das plântulas dentro do limite amostral foi aleatória ao longo da área de estudo priorizando a diversidade morfológica dos indivíduos. A fim de obter material herborizado e por não ser possível identificar todas as espécies em campo, no máximo três plântulas de cada morfoespécie foram coletadas para cultivo e posterior identificação. Adicionalmente, foram coletadas sementes e frutos a fim de obter mais plântulas da área de estudo.

As plântulas foram retiradas do solo com o auxílio de uma pá de jardinagem para evitar danos às raízes. Os torrões de terra foram removidos para facilitar o transporte e foram colocadas em recipientes com água até o transplante no viveiro (Viani \& Rodrigues 2007), localizado no Centro de Ciências Agrárias da Universidade Federal de São Carlos (UFSCar, campus Araras). Os recipientes definitivos para o transplante das plântulas foram tubetes de polipropileno de $20 \mathrm{~cm}$ de altura por $5 \mathrm{~cm}$ de diâmetro para acomodar as raízes que tinham seu comprimento semelhante. Raízes tortas ou maiores que os recipientes foram podadas para serem acomodadas corretamente nos recipientes sem serem dobradas ou quebradas (Viani $\&$ Rodrigues 2009). Foi utilizada terra como substrato e os tubetes ficaram em suportes com capacidade de 54 tubetes sobre o chão do viveiro, sob sombrite $50 \%$, com irrigação por aspersor quatro vezes ao dia por dois minutos e adubação líquida quinzenal. Durante o acompanhamento das plântulas no viveiro, os tubetes passaram por controle manual de ervas daninhas.

Sementes coletadas no solo e nas árvores da área de estudo foram levadas para germinação no viveiro da UFSCar campus Araras para obtenção de plântulas. Para as sementes médias e grandes foi adotada a semeadura direta em tubetes de polipropileno (Barbosa et al. 2013), tendo como substrato a terra ao redor do viveiro; foram colocadas folhas secas no fundo do tubete para diminuição da perda de terra com a irrigação. Com as sementes pequenas foi realizada a semeadura indireta em bandejas de plástico com areia grossa. A quebra da dormência das sementes com tegumento impermeável foi realizada através da escarificação mecânica (Barbosa et al. 2013).

Indivíduos de plântulas foram herborizados para posterior identificação e as exsicatas foram depositadas no herbário da UFSCar, campus Araras. Para a identificação das plântulas foram utilizados guias de identificação de espécies arbóreas, tais como Lorenzi (1992, 1998, 2009), Viani (2007), Barbosa et al. (2013) e Frigieri et al. (2016).
Adicionalmente, foram visitados os viveiros Pantanal Plantas (Município de Casa Branca, SP), Flora Planta Verde e o Viveiro Municipal do Centro de Educação Ambiental Pessegueiro (ambos no Município de Santa Cruz das Palmeiras, SP). A visualização e captura de imagens detalhadas das plântulas para identificação foi feita através da Lupa Digital USB Zoom 1000x.

As informações sobre a altura que os indivíduos de cada espécie podem alcançar foram extraídas de Matos et al. (2018), sendo as informações ausentes complementadas através de livros de identificação botânica (Bernacci \& Jung-Mendaçolli 2005, Lorenzi 2009, Lorenzi et al. 2009) e Flora do Brasil (2020 em construção). A fim de associar as espécies com os estratos que os indivíduos podem ocupar quando adultos, admitiu-se a altura referente aos estratos da floresta de acordo com Haddad et al. (2016) e a altura dos indivíduos segundo Matos et al. (2018). As espécies que possuíam altura fora do período de cada estrato foram inseridas para o estrato que estava com os valores mais próximos, a exemplo de Geissanthus ambiguus (Mart.) G.Agostini com altura entre 1 e $6 \mathrm{~m}$, que foi considerada como espécie de sub-bosque (8 a $10 \mathrm{~m}$ ), assim como Cariniana legalis (Mart.) Kuntze com 35 a 50 m, que foi inserida no estrato emergente $(20$ a $30 \mathrm{~m})$. As espécies que possuíam limite de altura máximo/mínimo igual ao valor mínimo/máximo de outro estrato (respectivamente), foram incluídas no outro estrato também, a exemplo de Dendropanax cuneatus (DC.) Decne. \& Planch., com altura mínima de $6 \mathrm{~m}$ e máxima de $20 \mathrm{~m}$, considerada espécie de sub-bosque, dossel ( 15 a $25 \mathrm{~m}$ ) e estrato emergente.

\section{Resultados e Discussão}

Foram coletados 116 indivíduos de 39 morfoespécies arbóreas, sendo 29 em nível de espécie, uma em nível de gênero, duas identificadas em nível de família e sete permaneceram indeterminadas. Foram obtidas por geminação de sementes um total de 40 espécies, das quais 13 também foram amostradas como plântulas, e 27 espécies foram obtidas exclusivamente por germinação de amostras de solo (tabela 1, figura 2).

Um estudo preliminar na mesma floresta ciliar da Guarnição da Aeronáutica de Pirassununga envolvendo a regeneração natural de indivíduos lenhosos entre 30 e $80 \mathrm{~cm}$ de altura (T. Hypolito, dados não publicados), identificou 49 espécies pertencentes a 21 famílias, as mais ricas sendo Myrtaceae (nove espécies), Meliaceae (seis espécies), Euphorbiaceae e Fabaceae (quatro espécies cada) (T. Hypolito, dados não publicados). O nosso estudo, que abrangeu espécies arbóreas obtidas por geminação de sementes no solo e coleta aleatória de plântulas entre 5 e $30 \mathrm{~cm}$ de altura amostrou mais espécies, sendo 56 espécies pertencentes a 27 famílias, sendo as mais ricas Fabaceae (16 espécies), Meliaceae (cinco espécies) e Rutaceae (quatro espécies). O mesmo estudo preliminar reconheceu apenas 14 (T. Hypolito, dados não publicados) das 56 espécies aqui identificadas (ou $25 \%$ do total), revelando que, embora usassem a mesma área de estudo, métodos diferentes permitem uma amostragem mais completa da biodiversidade local, especialmente de plântulas de espécies arbóreas. 
Tabela 1. Lista de plântulas de espécies arbóreas coletadas e/ou semeadas a partir de coletas realizadas na Guarnição da Aeronáutica de Pirassununga, Pirassununga, SP, Brasil. Forma de obtenção (FO). P: plântula coletada; S: semeadura. Altura estimada (AE) em metros (m). Ocorrência em fitofisionomias segundo a Flora do Brasil 2020 em construção (OF). Floresta Ciliar (FC); Floresta Estacional Semidecidual (FES). Categoria de ameaça segundo a Flora do Brasil 2020 em construção (CA). NA: não avaliada; pouco preocupante (PP), vulnerável (VU), quase ameaçada (QA) e em perigo (EP).

Table 1. List of seedlings of tree species collected and /or sown from collections carried out at the Guarnição da Aeronátuca de Pirassununga, Pirassununga, São Paulo State, Brazil. Method of production(FO). P: seedling collected; S: sowing. Estimated height (AE) in meters (m). Occurrence in phytophysiognomies according to Flora do Brasil 2020 under construction (OF). Riparian Forest (FC); Seasonal Semideciduous Forest (FES). Threat category according to Flora do Brasil 2020 under construction (CA). NA: Data Deficient; least concern (PP), vulnerable (VU), near threatened (QA) and endangered (EP).

\begin{tabular}{|c|c|c|c|c|}
\hline Famílias / Espécies & FO & $\mathrm{AE}(\mathrm{m})$ & $\mathrm{OF}$ & $\mathrm{CA}$ \\
\hline \multicolumn{5}{|l|}{ Anacardiaceae } \\
\hline Astronium graveolens Jacq. & $\mathrm{P}$ & $15-25$ & $\mathrm{FC} / \mathrm{FES}$ & $\mathrm{PP}$ \\
\hline \multicolumn{5}{|l|}{ Apocynaceae } \\
\hline Aspidosperma cylindrocarpon Müll.Arg. & $\mathrm{P} / \mathrm{S}$ & $6-30$ & $\mathrm{FC} / \mathrm{FES}$ & $\mathrm{PP}$ \\
\hline \multicolumn{5}{|l|}{ Araliaceae } \\
\hline Dendropanax cuneatus (DC.) Decne. \& Planch. & S & $6-20$ & $\mathrm{FC} / \mathrm{FES}$ & $\mathrm{PP}$ \\
\hline Schefflera sp. & $\mathrm{P}$ & - & - & - \\
\hline \multicolumn{5}{|l|}{ Arecaceae } \\
\hline Acrocomia aculeata (Jacq.) Lodd. ex Mart. & S & $10-15$ & $\mathrm{FC} / \mathrm{FES}$ & NA \\
\hline Euterpe edulis Mart. & $\mathrm{P} / \mathrm{S}$ & $5-12$ & $\mathrm{FC}$ & $\mathrm{VU}$ \\
\hline \multicolumn{5}{|l|}{ Bignoniaceae } \\
\hline Handroanthus impetiginosus (Mart. ex DC.) Mattos & S & $8-12$ & - & QA \\
\hline \multicolumn{5}{|l|}{ Cactaceae } \\
\hline Pereskia grandifolia Haw. & $\mathrm{P}$ & $2-10$ & $\mathrm{FC} / \mathrm{FES}$ & $\mathrm{PP}$ \\
\hline \multicolumn{5}{|l|}{ Clusiaceae } \\
\hline Garcinia gardneriana (Planch. \& Triana) Zappi & $\mathrm{S}$ & 10 & $\mathrm{FC} / \mathrm{FES}$ & NA \\
\hline \multicolumn{5}{|l|}{ Combretaceae } \\
\hline Combretum leprosum Mart. & $\mathrm{P} / \mathrm{S}$ & $10-15$ & FES & NA \\
\hline \multicolumn{5}{|l|}{ Euphorbiaceae } \\
\hline Actinostemon klotzschii (Didr.) Pax & $\mathrm{P}$ & $1-9$ & $\mathrm{FC}$ & NA \\
\hline Croton floribundus Spreng. & $\mathrm{S}$ & $6-15$ & $\mathrm{FC} / \mathrm{FES}$ & NA \\
\hline Croton urucurana Baill. & S & $7-14$ & $\mathrm{FC} / \mathrm{FES}$ & NA \\
\hline \multicolumn{5}{|l|}{ Fabaceae } \\
\hline Albizia niopoides (Spruce ex Benth.) Burkart & $\mathrm{P}$ & $10-22$ & $\mathrm{FC} / \mathrm{FES}$ & $\mathrm{PP}$ \\
\hline Bauhinia longifolia (Bong.) Steud. & $\mathrm{P}$ & $4-7$ & $\mathrm{FC} / \mathrm{FES}$ & NA \\
\hline Copaifera langsdorffii Desf. & $\mathrm{P} / \mathrm{S}$ & $10-15$ & $\mathrm{FC} / \mathrm{FES}$ & NA \\
\hline Centrolobium tomentosum Guillem. ex Benth. & $\mathrm{P} / \mathrm{S}$ & $10-22$ & FES & $\mathrm{PP}$ \\
\hline Enterolobium contortisiliquum (Vell.) Morong & S & $20-35$ & $\mathrm{FC} / \mathrm{FES}$ & NA \\
\hline Erythrina falcata Benth. & $\mathrm{S}$ & $20-30$ & $\mathrm{FC} / \mathrm{FES}$ & NA \\
\hline Holocalyx balansae Micheli & $\mathrm{P}$ & $15-25$ & FES & NA \\
\hline Hymenaea courbaril L. & $\mathrm{P} / \mathrm{S}$ & $15-20$ & $\mathrm{FC}$ & $\mathrm{PP}$ \\
\hline Inga marginata Willd. & $\mathrm{P}$ & $5-20$ & $\mathrm{FC} / \mathrm{FES}$ & NA \\
\hline Inga vera subsp. affinis (DC.) T.D.Penn. & $\mathrm{S}$ & $5-10$ & $\mathrm{FC} / \mathrm{FES}$ & NA \\
\hline Lonchocarpus latifolius (Willd.) DC. & $\mathrm{P}$ & $4-8$ & FC & NA \\
\hline Myroxylon peruiferum L.f. & $\mathrm{P} / \mathrm{S}$ & $12-26$ & $\mathrm{FC} / \mathrm{FES}$ & $\mathrm{PP}$ \\
\hline Ormosia arborea (Vell.) Harms & $\mathrm{S}$ & $15-20$ & - & NA \\
\hline Piptadenia gonoacantha (Mart.) J.F.Macbr. & $\mathrm{P}$ & $10-20$ & $\mathrm{FC} / \mathrm{FES}$ & $\mathrm{PP}$ \\
\hline Platycyamus regnellii Benth. & $\mathrm{S}$ & $15-22$ & $\mathrm{FC} / \mathrm{FES}$ & NA \\
\hline Senegalia polyphylla (DC.) Britton \& Rose & $\mathrm{S}$ & $15-20$ & $\mathrm{FC} / \mathrm{FES}$ & NA \\
\hline
\end{tabular}




\begin{tabular}{|c|c|c|c|c|}
\hline Famílias / Espécies & FO & $\mathrm{AE}(\mathrm{m})$ & $\mathrm{OF}$ & CA \\
\hline \multicolumn{5}{|l|}{ Lauraceae } \\
\hline Nectandra megapotamica (Spreng.) Mez & $\mathrm{P}$ & 22 & FC/FES & NA \\
\hline \multicolumn{5}{|l|}{ Lecythidaceae } \\
\hline Cariniana estrellensis (Raddi) Kuntze & $\mathrm{P} / \mathrm{S}$ & $35-45$ & FES & NA \\
\hline Cariniana legalis (Mart.) Kuntze & $\mathrm{P}$ & $35-50$ & - & $\mathrm{EP}$ \\
\hline \multicolumn{5}{|l|}{ Magnoliaceae } \\
\hline Magnolia ovata (A.St.-Hil.) Spreng. & $\mathrm{S}$ & $20-30$ & $\mathrm{FC}$ & $\mathrm{PP}$ \\
\hline \multicolumn{5}{|l|}{ Malvaceae } \\
\hline Luehea divaricata Mart. \& Zucc. & $\mathrm{S}$ & $5-25$ & $\mathrm{FC}$ & NA \\
\hline Pseudobombax grandiflorum (Cav.) A.Robyns & $\mathrm{S}$ & $15-25$ & FES & PP \\
\hline \multicolumn{5}{|l|}{ Meliaceae } \\
\hline Cedrela fissilis Vell. & $\mathrm{S}$ & $8-30$ & FES & $\mathrm{VU}$ \\
\hline Guarea guidonia (L.) Sleumer & $\mathrm{S}$ & $3-20$ & $\mathrm{FC}$ & NA \\
\hline Trichilia catigua A.Juss. & $\mathrm{P} / \mathrm{S}$ & 10 & $\mathrm{FC} / \mathrm{FES}$ & NA \\
\hline Trichilia hirta L. & S & 8 & FC/FES & PP \\
\hline Trichilia pallida $\mathrm{Sw}$. & $\mathrm{S}$ & 7 & $\mathrm{FC} / \mathrm{FES}$ & NA \\
\hline \multicolumn{5}{|l|}{ Moraceae } \\
\hline Ficus adhatodifolia Schott in Spreng. & S & $8-12$ & $\mathrm{FC} / \mathrm{FES}$ & NA \\
\hline Ficus guaranitica Chodat & S & $10-20$ & FES & NA \\
\hline \multicolumn{5}{|l|}{ Myrtaceae } \\
\hline Syzygium jambos (L.) Alston & $\mathrm{S}$ & $10-15$ & - & - \\
\hline Myrtaceae sp 1. & $\mathrm{P}$ & - & - & - \\
\hline Myrtaceae sp 2. & $\mathrm{P}$ & - & - & - \\
\hline \multicolumn{5}{|l|}{ Phyllanthaceae } \\
\hline Savia dictyocarpa Müll.Arg. & $\mathrm{S}$ & $3-18$ & - & PP \\
\hline \multicolumn{5}{|l|}{ Primulaceae } \\
\hline Geissanthus ambiguus (Mart.) G.Agostini & $\mathrm{P} / \mathrm{S}$ & $1-6$ & - & NA \\
\hline \multicolumn{5}{|l|}{ Proteaceae } \\
\hline Roupala montana var. brasiliensis (Klotzsch) K.S.Edwards & $\mathrm{P}$ & $15-25$ & $\mathrm{FC}$ & NA \\
\hline \multicolumn{5}{|l|}{ Rhamnaceae } \\
\hline Rhamnidium elaeocarpum Reissek & $\mathrm{S}$ & $4-9$ & $\mathrm{FC} / \mathrm{FES}$ & NA \\
\hline \multicolumn{5}{|l|}{ Rosaceae } \\
\hline Prunus myrtifolia (L.) Urb. & $\mathrm{P} / \mathrm{S}$ & $8-15$ & - & NA \\
\hline \multicolumn{5}{|l|}{ Rubiaceae } \\
\hline Psychotria carthagenensis Jacq. & $\mathrm{P}$ & $3-12$ & $\mathrm{FC} / \mathrm{FES}$ & NA \\
\hline \multicolumn{5}{|l|}{ Rutaceae } \\
\hline Esenbeckia febrifuga (A.St.-Hil.) A. Juss. ex Mart. & $\mathrm{P} / \mathrm{S}$ & $1-11$ & FES & NA \\
\hline Esenbeckia grandiflora Mart. & $\mathrm{P}$ & $4-7$ & $\mathrm{FC} / \mathrm{FES}$ & NA \\
\hline Galipea jasminiflora (A.St.-Hil.) Engl. & $\mathrm{P}$ & $2-7$ & FES & NA \\
\hline Metrodorea nigra A.St.-Hil. & S & $4-8$ & $\mathrm{FC} / \mathrm{FES}$ & NA \\
\hline \multicolumn{5}{|l|}{ Sapindaceae } \\
\hline Allophylus racemosus $\mathrm{Sw}$. & $\mathrm{P}$ & $3-10$ & $\mathrm{FC} / \mathrm{FES}$ & NA \\
\hline \multicolumn{5}{|l|}{ Sapotaceae } \\
\hline Chrysophyllum gonocarpum (Mart. \& Eichler ex Miq.) Engl. & $\mathrm{P} / \mathrm{S}$ & $10-20$ & FES & NA \\
\hline \multicolumn{5}{|l|}{ Urticaceae } \\
\hline Cecropia pachystachya Trécul & $\mathrm{S}$ & $2,5-12$ & $\mathrm{FC} / \mathrm{FES}$ & NA \\
\hline \multicolumn{5}{|l|}{ Verbenaceae } \\
\hline Citharexylum myrianthum Cham. & $\mathrm{S}$ & $8-20$ & FC/FES & NA \\
\hline
\end{tabular}



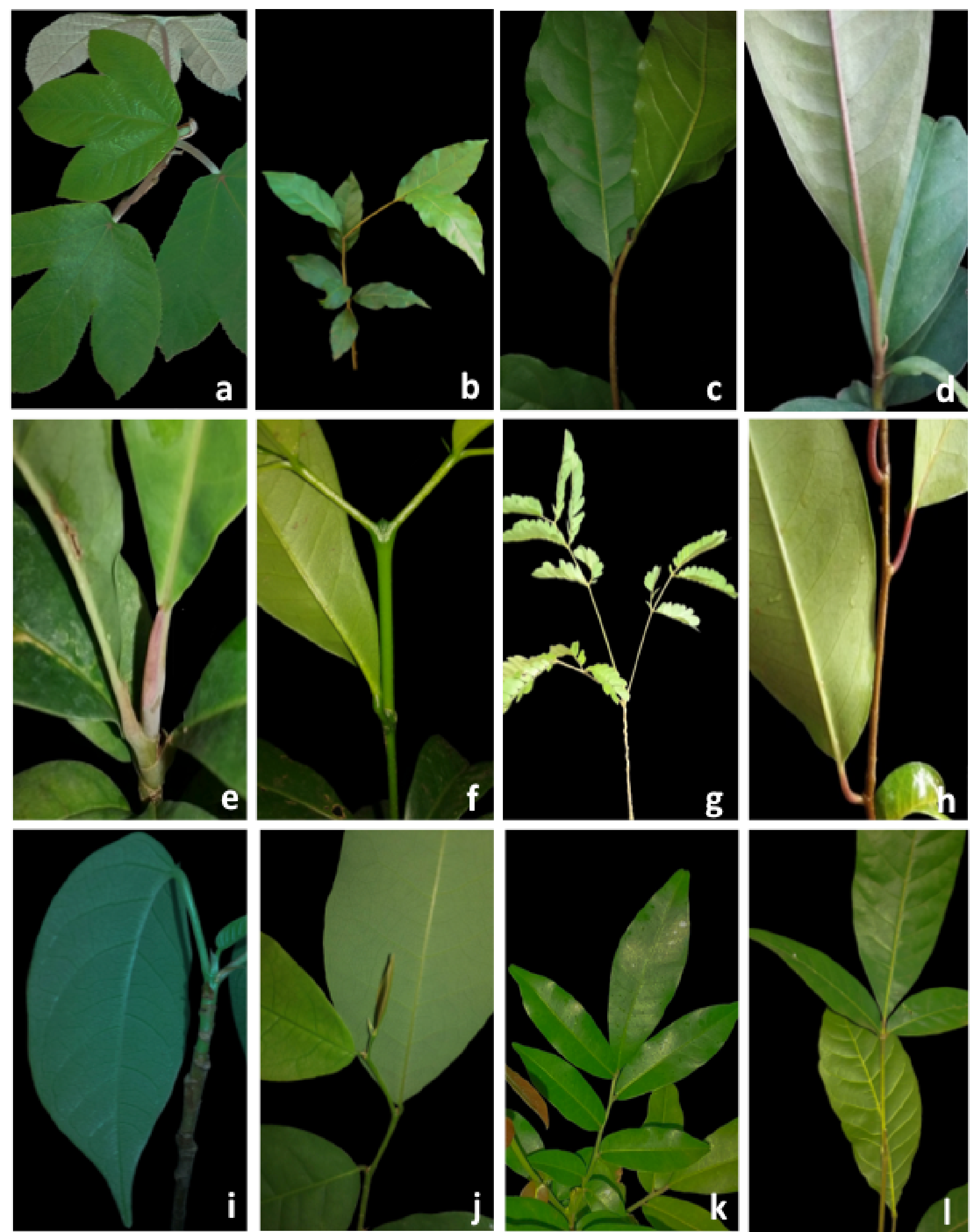

Figura 2. Detalhes das plântulas da floresta ciliar da Guarnição da Aeronáutica de Pirassununga, SP, Brasil. a. Cecropia paschystachya Trécul. b e c. Combretum leprosum Mart. d. Geissanthus ambiguus (Mart.) G.Agostini. e. Magnolia ovata (A.St.-Hil.) Spreng. f. Metrodorea nigra A.St.-Hil. g. Piptadenia gonoacantha (Mart.) J.F.Macbr. h. Prunus myrtifolia (L.) Urb. i. Pseudobombax grandiflorum (Cav.) A.Robyns. j. Savia dictyocarpa Mull.Arg. k. Trichilia catigua A.Juss. 1. Trichilia pallida Sw.

Figure 2. Details of the seedlings found at the riparian forest of the Guarnição da Aeronáutica de Pirassununga, São Paulo State, Brazil. a. Cecropia paschystachya Trécul. b e c. Combretum leprosum Mart. d. Geissanthus ambiguus (Mart.) G.Agostini. e. Magnolia ovata (A.St.-Hil.) Spreng. f. Metrodorea nigra A.St.-Hil. g. Piptadenia gonoacantha (Mart.) J.F.Macbr. h. Prunus myrtifolia (L.) Urb. i. Pseudobombax grandiflorum (Cav.) A.Robyns. j. Savia dictyocarpa Mull.Arg. k. Trichilia catigua A.Juss. 1. Trichilia pallida Sw. 
Dentre as espécies de plântulas aqui reconhecidas, 23 espécies, embora de ocorrência conhecida para o Estado de São Paulo, nunca foram relatadas em florestas ciliares (Flora do Brasil 2020 em construção). No entanto, trabalhos com árvores e plântulas dentro da bacia do Rio Mogi Guaçu já haviam amostrado algumas dessas espécies. Aqui nós citamos oito novas ocorrências para a floresta cilar: $C$. fissilis, C. tomentosum, C. leprosum, Esenbeckia febrifuga (A.St.-Hil.) A.Juss. ex Mart., G. ambiguus, H. balansae, Ormosia arborea (Vell.) Harms e P. grandiflorum. Essas espécies eram anteriormente conhecidas em floresta estacional semidecidual. Finalmente, também amostramos Handroanthus impetiginosus (Mart. ex DC.) Mattos pela primeira vez em floresta ciliar, espécie que também nunca foi relatada em floresta estacional semidecidual.

De acordo com a Lei ${ }^{\circ} 12.651$ de 25 de maio de 2012 (Brasil 2012), no seu Artigo $4^{\circ}$ inciso I, considera-se que as Áreas de Preservação Permanentes (APP) urbanas ou rurais são as faixas marginais de qualquer curso d'água natural perene e intermitente, desde a borda da calha do leito regular, em largura mínima de 100 metros para os cursos d'água entre de 50 e $200 \mathrm{~m}$ de largura. Considerando essa metragem, toda a área de estudo do Rio Mogi Guaçu está inserida dentro da APP ciliar e, portanto, a partir do presente trabalho, é possível destacar a ocorrências dessas nove espécies citadas à fitofisionomia de floresta ciliar para o Estado de São Paulo e a necessidade de estudar o interior do fragmento de floresta estacional semidecidual, a fim de evidenciar o quanto a influência entre as fitofisionomias é recíproca, e também o quanto a definição sobre o que é uma floresta ciliar é necessária.

Além disso, outras espécies como C. leprosum, Garcinia gardneriana (Planch. \& Triana) Zappi, Lonchocarpus latifolius (Willd.) DC. e P. grandifolia não foram citadas em trabalhos anteriores envolvendo o estrato arbóreo na região de Pirassununga, dentro dos limites da UGRHI de Mogi Guaçu (Bertoni 1984, Silva \& Soares 2002, 2003, Nóbrega et al. 2007, Sartori et al. 2007, Aquino \& Barbosa 2009, Sartori 2010, Toledo Filho et al. 2010, Naves 2013, Queiroz et al. 2017). Dentre essas espécies, C. leprosum está sendo relatada pela primeira vez no Estado de São Paulo (Flora do Brasil 2020 em construção).

Combretum leprosum pode crescer como árvore ou trepadeira, que ocorre no Paraguai e no Brasil. No Brasil, foi amostrada nas regiões norte, nordeste, centro-oeste e sudeste, nas fitofisionomias de caatinga stricto sensu, carrasco, cerrado latu sensu, floresta estacional decidual e floresta estacional semidecidual, entretanto não é conhecida nos Estados do Rio de Janeiro e São Paulo (Flora do Brasil 2020 em construção), ou em estudos dedicados às trepadeiras do Estado de São Paulo (Hora \& Soares 2002, Udulutsch et al. 2004, Tibiriçá et al. 2006, Oliveira 2012, Romaniuc Neto et al. 2012, Dinato 2014. Contudo, existe uma coleção da espécie para o Estado de São Paulo no SpeciesLink (2020): A.P. Viegas 5117 (ESA), que foi coletada em maio de 1939. Nós não localizamos indivíduos adultos, mas encontramos plântulas e frutos sobre a serapilheira.
Considerando que entre as ações prioritárias para a conservação da flora presente na UGRHI de Mogi Guaçu e outras áreas similares, atenção deve ser dada à elaboração de inventários biológicos contínuos (Durigan et al. 2008), e que em florestas de grande porte onde não é possível encontrar o indivíduo adulto ou ter acesso a copa, estudar as plântulas é uma ferramenta importante para a identificação de espécies, como mostrado nesse trabalho.

A presença de espécies invasoras e exóticas em fragmentos de florestas nativas tem sido mostrada por vários autores: Silva Junior (2002, Coffea arabica L.), Araujo et al. (2004, Melia azedarach L.), Aquino \& Barbosa (2009), Moreira \& Carvalho (2013) e Durães et al. (2014, que levantaram em relação à Syzygium jambos (L.) Alston, Pinus elliottii Engelm e Mangifera indica L.), dentre outros. O jambo ( $S$. jambos), uma espécie frutífera, foi utilizado na arborização da Guarnição da Aeronáutica de Pirassununga, é uma das três espécies exóticas anteriormente encontradas na área de estudo (T. Hypolito, dados não publicados). No presente estudo, essa espécie foi repertoriada como sementes que, em viveiro, geraram plântulas viáveis. Sementes de $S$. jambos devem ter alcançado a floresta ciliar através de animal dispersor, como, por exemplo, os macacosprego (Sapajus sp., Cebidae). Isso é preocupante, já que características dessa espécie, como a plasticidade fenotípica e genotípica, tolerância à sombra, rápida germinação, crescimento das mudas e reprodução precoce favorecem o aumento da população, juntamente com dispersão efetiva tornam-na uma espécie invasora que pode comprometer a diversidade local, e até acelera o processo de extinção de espécies nativas (Carvalho 2005, Colmanetti \& Barbosa 2013). Por isso, estudos de monitoramento de fragmentos florestais são essenciais, a fim de obter informações para orientar ações que garantam a recuperação e a conservação desses fragmentos (Silva Junior 2002), bem como acompanhar a população das espécies invasoras que exigem atenção específica (Colmanetti \& Barbosa 2013).

Quanto ao grau de ameaça e possibilidade de extinção, das 55 espécies nativas identificadas, 38 ainda não foram avaliadas, 13 estão da categoria pouco preocupante, duas são consideradas vulneráveis, uma é considerada quase ameaçada e uma é considerada em perigo de extinção (Flora do Brasil 2020 em construção), ou seja, quatro espécies reconhecidas no presente estudo estão sob algum grau de ameaça. A maioria das espécies não foi avaliada, o que não significa que estejam fora de perigo, até porque a contínua fragmentação de formações vegetais leva à destruição de habitats e extinção de muitas espécies da fauna e flora que nem chegam a ter suas ocorrências reconhecidas (Eisenlohr et al. 2015). Na UGRHI de Mogi Guaçu há ocorrência de apenas 16 Unidades de Conservação e outras áreas especialmente protegidas, das quais duas unidades são Áreas de Proteção Ambiental e apenas quatro Unidades de Conservação com área igual ou superior a 1.500 ha (Xavier et al. 2008) as quais, juntamente com a área do presente estudo, são imprescindíveis à conservação da biodiversidade da Mata Atlântica, um dos hotspots para conservação da biodiversidade mundial (Myers et al. 2000). 
Variação da composição dos estratos da floresta ciliar A família Fabaceae é normalmente a predominante nos estratos superiores das florestas ciliares (Rodrigues et al. 2010). Esta família foi a mais rica em número de espécies no presente estudo (16 espécies), assim como teve o maior número de espécies que podem ocupar o dossel (13 espécies) e o estrato emergente (12 espécies). Em florestas tropicais, onde a estratificação vertical nem sempre é nítida, conhecer as espécies presentes pode auxiliar na definição desses estratos (Rodrigues et al. 2010). Seguindo a definição de estratos indicada por Haddad et al. (2016), na qual o sub-bosque estaria entre $8-10 \mathrm{~m}$, o dossel entre $15-25 \mathrm{~m}$ e as árvores emergente atingiriam entre 20 e 30 $\mathrm{m}$, espécies aqui identificadas que ocupam o sub-bosque, seriam tais como Pereskia grandifolia Haw. (2-10 m de altura), T. catigua $(10 \mathrm{~m})$ e Allophylus racemosus Sw. (3$10 \mathrm{~m})$; no dossel, encontraríamos Astronium graveolens Jacq. (15-25 m), H. balansae (15-25 m) e Nectandra megapotamica (Spreng.) Mez (22 m); e emergentes seriam E. contortisiliquum (20-35 m), Cariniana estrellensis (Raddi) Kuntze (35-45 m) e M. ovata (20-30 m). Há, ainda, espécies com potencial para ocupar mais de um estrato, como Aspidosperma cylindrocarpon Müll.Arg. (6-30 m) ou Cedrela fissilis Vell. (8-30 m). O banco de plântulas é um indicador do potencial regenerativo das espécies em uma floresta (Higuchi 2003), e as espécies aqui amostradas tem possibilidade de ocupar diferentes estratos, sendo possível reconhecer o potencial da estratificação da floresta na sua manutenção ao longo da sucessão ecológica. Estratificação é um fator que pode ajudar a sustentar uma fauna mais variada, proporcionando-lhe alimento e abrigo, que por sua vez ajuda a própria manutenção de uma variedade de espécies, uma vez que a morfologia e altura das copas interfere na seleção das sementes a serem dispersas pelos dispersores funcionalmente diferentes, como saguis em árvores de pequeno e médio porte e macacos maiores ou grandes aves frugívoras em árvores de médio a grande porte (Clark \& Poulsen 2001).

As características morfológicas das plântulas podem apresentar diferenças em relação aos adultos (Higuchi 2003, Alves \& Metzger 2006, Higuchi et al. 2006, Frigieri et al. 2016). Algumas espécies na fase adulta possuem folhas simples, enquanto que na fase de plântulas apenas as têm em menor tamanho (exemplo: Chrysophyllum gonocarpum (Mart. \& Eichler ex Miq.) Engl., Psychotria carthagenensis Jacq. e Savia dictyocarpa Müll.Arg., figura 2j). Outras possuem as folhas alterna até cerca de $15 \mathrm{~cm}$, depois as folhas novas são opostas, assim como as dos indivíduos adultos (Rhamnidium elaeocarpum Reissek e Combretum leprosum Mart., figura 2 b e c). Na família Urticaceae a nervação das folhas pode ser actinódroma e raramente peninérvea, lâmina inteira ou palmatilobada; para o gênero Cecropia Loefl., é citada a lâmina peltada palmatilobada, palminérvea nas folhas jovens e radial nas adultas (Gaglioti \& Romaniuc Neto 2012). Aqui nós relatamos que as plântulas com até cerca de $20 \mathrm{~cm}$ de Cecropia pachystachya Trécul (figura 2 a) têm folhas inteiras, depois começam a apresentar três lobos, com o número de lobos aumentando gradativamente conforme a plântula cresce.
Também notamos que nessa espécie a nervação em plântulas pode ser peninérvea quando não apresenta lobos e palmatinérvea quando lobada; na ausência de lobos a inserção do pecíolo no limbo é na extremidade basal, na presença gradual dos lobos fica cada vez mais nítida a inserção do pecíolo no meio do limbo, caracterizando-a como folha peltada.

Algumas espécies apresentam plântulas com folhas simples, que depois de atingirem certa altura passam a apresentar folhas compostas semelhantes às plantas adultas, como em Trichilia pallida Sw. (figura 2 1) e Metrodorea nigra A.St.-Hil. (figura $2 \mathrm{f})(25 \mathrm{~cm})$; Pseudobombax grandiflorum (Cav.) A.Robyns (figura 2i) e Guarea guidonia (L.) Sleumer $(20 \mathrm{~cm})$; Centrolobium tomentosum Guillem. ex Benth. (15 cm); Trichilia catigua A.Juss. (10 $\mathrm{cm}$ ) (figura $2 \mathrm{k}$ ). Em outras espécies, as primeiras folhas após os cotilédones já se apresentam compostas, mas com menos folíolos que nas folhas dos indivíduos adultos, tais como em Inga marginata Willd. e Inga vera subsp. affinis (DC.) T.D.Penn. Já em Holocalyx balansae Micheli e Erythrina falcata Benth., as folhas das plântulas eram muito semelhantes às dos indivíduos adultos. Em plântulas de C. tomentosum, as primeiras folhas são simples e as seguintes compostas, aumentando-se o número de folíolos conforme novas folhas vão surgindo, porém o folíolo terminal da folha composta e as primeiras folhas simples são semelhantes quanto à forma, margem, tipo de base e ápice foliar.

Embora existam chaves de identificação e descrição detalhada para as espécies do gênero Schefflera J.R. Forst. \& G. Forst. em Fiaschi \& Pirani (2007) e Fiaschi et al. (2007), não foi possível identificar a espécie do gênero aqui reconhecido, já que a combinação de ápice, base e indumento dos folíolos eram incompatíveis com as descrições das espécies desse gênero para o Estado de São Paulo. A espécie nativa Didymopanax vinosus (Cham. \& Schltdl.) Marchal, (da qual Schefflera vinosa (Cham. \& Schltdl.) Frodin \& Fiaschi é sinônimo), e a espécie exótica Dendropanax arboreus (L.) Decne. \& Planch. (da qual Schefflera arborea (L.) M.Gómez é sinônimo), foram as mais próximas, mas a maleabilidade, brilho e indumento dos folíolos da plântula a distingue dessas duas espécies. Apenas quando a plântula atingir o estágio juvenil ou adulto será possível averiguar se essas características se manterão a fim de certificar que seja uma espécie exótica, nativa já identificada ou mesmo uma nova espécie ou nova ocorrência para o Estado de São Paulo.

Embora os estudos de Filardi et al. (2016) e de Tamashiro \& Escobar (2016) contenham a descrição de Enterolobium contortisiliquum (Vell.) Morong. e Piptadenia gonoacantha (Mart.) J.F.Macbr. (figura 2g), características importantes que auxiliaram na distinção entre ambas, como a presença de lenticelas em $E$. contortisiliquum e pequenos acúleos ao longo do pecíolo e raque em $P$. gonoacantha não foram citados, provavelmente pelo fato de que nas primeiras folhas os acúleos são inconspícuos, sendo mais perceptíveis pelo tato. Segundo Souza Júnior e Brancalion (2016), ocorre a presença de espinhos no caule de $P$. gonoacantha na fase de muda, o que também foi observado no presente estudo. 
Como são utilizadas na identificação das plântulas características vegetativas, alguns detalhes tais como formato do nectário, tipo de base e ápice dos foliólulos, indumento do caule, distribuição dos tricomas e nervuras; são características indispensáveis para a confirmação da identificação das espécies aqui reconhecidas, a exemplo de $E$. contortisiliquum e $P$. gonoacantha.

Esser (1999) traz detalhes vegetativos importantes para a identificação das espécies de Maprounea, como a presença de glândulas próximas da base das folhas, possibilitando a exclusão de $M$. guianensis Aubl. como candidata à espécie de uma das plântulas que permaneceu sem identificação; havia grande semelhança sobre dimensões de pecíolo e folha, tipo de base e ápice foliar além das nervuras, mas nenhuma das folhas da plântula em questão possuía glândulas. Em outros casos, algumas características próximas à base da folha auxiliaram na identificação das espécies, como o par de glândulas na face abaxial de Prunus myrtifolia (L.) Urb. (Figura $2 \mathrm{~h}$ ), as diminutas pintas vináceas em toda a face abaxial de Geissanthus ambiguus (Mart.) G.Agostini e as expanções laterais do pecíolo de Magnolia ovata (A.St.Hil.) Spreng. que possibilitou diferenciar as duas últimas (figura $2 \mathrm{~d}$ e e).

Uma das espécies de Ficus indicada no presente estudo também foi de difícil identificação, devido à proximidade dos caracteres morfológicos entre as espécies e até às divergências entre autores quanto ao nome correto a ser considerado. Com base em caracteres morfológicos, inicialmente o indivíduo encontrado foi identificado como Ficus insipida Willd., presente somente na região amazônica (Flora do Brasil 2020 em construção). Mas, Mendonça-Souza (2006) propõe que seja feita a sinonimização de Ficus adhatodifolia Schott in Spreng. em F. insipida, o que não foi de fato publicado, já que esses nomes continuam sendo válidos e a Flora do Brasil 2020 em construção mantém a distinção entre elas. Assim, no presente estudo optou-se por associar o indivíduo ao nome $F$. adhatodifolia, já que esta espécie apresenta ampla distribuição no Brasil (Flora do Brasil 2020 em construção), incluindo o Estado de São Paulo.

Como as plântulas de espécies arbóreas, arbustivas e trepadoras são semelhantes quando muito pequenas (ca. $10 \mathrm{~cm}$ ), é fácil serem confundidas quanto ao hábito. Como visto acima, algumas espécies arbóreas apresentam características diferentes dos presentes nos indivíduos adultos quando muito pequenas, e para aquelas não pioneiras, com crescimento lento no caso das plântulas, recomendando, para melhor identificação das plântulas um acompanhamento mais longo em viveiro, incluindo registro fotográfico no intervalo de 20 a $30 \mathrm{~cm}$ de altura, para conferir se suas características morfológicas inicialmente constatadas serão mantidas.

\section{Conclusões}

O fragmento florestal estudado está em uma área militar e abriga espécies raras e com diferentes graus de ameaça, além de novas ocorrências para bacia do Rio Mogi Guaçu e até para o Estado de São Paulo. A vegetação na área de estudo pode manter-se estratificada ao longo da sucessão florestal e é muito possível que estabeleça conexão com outros fragmentos de outros municípios; o que conferem à Guarnição da Aeronáutica de Pirassununga um papel relevante para a conservação da biodiversidade no Estado de São Paulo. O presente estudo mostra a necessidade da descrição de vários aspectos morfológicos para a correta identificação e distribuição de plântulas de espécies arbóreas em área de floresta ciliar, fitofisionomia altamente ameaçada no Estado de São Paulo. Estudos envolvendo a diversidade e a morfologia de plântulas devem ser realizados em outros locais, a fim de favorecerem o reconhecimento de padrões quanto ao comportamento, ocorrência e diversidade de espécies, contribuindo para estratégias de conservação.

\section{Agradecimentos}

Os autores agradecem à Guarnição da Aeronáutica de Pirassununga, pela autorização e apoio para a realização do presente estudo; ao Laboratório de Silvicultura e Pesquisas Florestais /UFSCar, pelo empréstimo do viveiro e do material para cultivo das plântulas; ao viveiros Pantanal Plantas, Flora Planta Verde e ao Viveiro Municipal do Centro de Educação Ambiental Pessegueiro, pela autorização da visitação e pelo acesso às mudas; e à Thelma Boerner Hypolito e à Emmanuélly Maria Souza Fernandes, pelas informações sobre a vegetação da Guarnição.

\section{Contribuição dos Autores}

Israel Henrique Buttner Queiroz: Substancial contribuição na delimitação, coleta e análise de dados do estudo, bem como autoria das fotografias, identificação das espécies e elaboração do manuscrito.

Ricardo Augusto Gorne Viani: Contribuições para o desenho da amostragem, identificação das espécies e revisão do manuscrito.

Renata Sebastiani: Contribuição na delimitação do estudo, na análise dos dados e na preparação e revisão do manuscrito.

\section{Conflito de interesse}

Não há conflito de interesse.

\section{Literatura citada}

Araujo, M.M., Longhi, S.J., Barros, P.L.C. \& Brena, D.A. 2004. Caracterização da chuva de sementes, banco de sementes do solo e banco de plântulas em Floresta Estacional Decidual ripária Cachoeira do Sul, RS, Brasil. Scientia Florestalis 66: 128-141.

Alves, L.F. \& Metzger, J.P. 2006. A regeneração florestal em áreas de floresta secundária na Reserva Florestal do Morro Grande, Cotia, SP. Biota Neotropica 6: 1-26.

Aquino, C. \& Barbosa, L.M. 2009. Classes sucessionais e síndromes de dispersão de espécies arbóreas e arbustivas existentes em vegetação ciliar remanescente (Conchal, SP), como subsídio para avaliar o potencial do fragmento como fonte de propágulos para enriquecimento de áreas revegetadas no rio Mogi-Guaçu, SP. Revista Árvore 33: $349-358$. 
Barbosa, L.M., Parajara, F.C. \& Barbosa, T.C. 2013. Manual de Orientação para Implantação de viveiro de mudas. Instituto de Botânica, São Paulo.

Batalha, M.A., Aragaki, S. \& Mantovani, W. 1997. Variações fenológicas das espécies do Cerrado em Emas (Pirassununga, SP). Acta Botanica Brasilica 11: 61-78.

Bernacci, L.C. \& Jung-Mendaçolli, S.L. 2005. Ardisia Sw. In: M.G.L. Wanderley, G.J. Shepherd, T.S. Melhem \& A.M. Giulietti (coords). Flora Fanerogâmica do Estado de São Paulo. FAPESP, RiMa, São Paulo, v. 4, pp. 280-283.

Bertoni, J.E.A. 1984. Composição Florística e Estrutura Fitossociológica de uma Floresta do Interior do Estado de São Paulo: Reserva Estadual de Porto Ferreira. Dissertação de Mestrado, Universidade Estadual de Campinas, Campinas.

Brasil. Lei n. ${ }^{\circ} 12.651$, de 25 de maio de 2012. Dispõe sobre a proteção da vegetação nativa; altera as Leis $n^{\circ} \mathrm{s} 6.938$, de 31 de agosto de 1981, 9.393, de 19 de dezembro de 1996, e 11.428, de 22 de dezembro de 2006; revoga as Leis $\mathrm{n}^{\mathrm{o}} \mathrm{s} 4.771$, de 15 de setembro de 1965, e 7.754, de 14 de abril de 1989, e a Medida Provisória no 2.16667 , de 24 de agosto de 2001; e dá outras providências. Diário Oficial da União, DF, 28 mai. 2012. Disponível em <https://www.jusbrasil.com.br/diarios/37352346/ dou-secao-1-28-05-2012-pg-1> (acesso em 10-IX-2020.

Cardoso-Leite, E., Covre, T.B., Ometto, R.G., Cavalcanti, D.C. \& Pagani, M.I. 2004. Fitossociologia e caracterização sucessional de um fragmento de mata ciliar, em Rio Claro/SP, como subsídio à recuperação da área. Revista do Instituto Florestal 16: 31-41.

Carvalho F.A. 2005. Syzygium jambos (L.) Alston - uma invasora na Mata Atlântica? Ministério do Meio Ambiente. Disponível em https:/www.mma.gov.br/ estruturas/174/_arquivos/174_05122008112733.pdf (acesso em 09-I-2020).

Clark, C.J. \& Poulsen, J.R. 2001. The role of arboreal seed dispersal groups on the seed rain of a lowland tropical forest. Biotropica 33: 606-620.

Colmanetti, M.A.A. \& Barbosa, L.M. 2013. Fitossociologia e estrutura do estrato arbóreo de um reflorestamento com espécies nativas em Mogi-Guaçu, SP, Brasil. Hoehnea 40: 419-435.

Dinato, D.O. 2014. Florística vascular não arbórea de um fragmento de floresta estacional semidecidual no município de Analândia - SP. Dissertação de Mestrado, UNESP, Rio Claro.

Durães, M.C.O., Sales, N.L., Ângelo Neto, S. \& Figueiredo, M.A.P. 2014. Levantamento florístico do estrato arbóreo de três fragmentos de floresta ciliar como subsídio à recomposição da vegetação do Rio Cedro, Montes Claros - MG. Ciência Florestal 24: 47-58.

Durigan, G., Mamede, M.C.H., Ivanaukas, N.M., Siqueira, M.F., Joly, C.A., Moura, C., Barros, F., Souza, F.M., Vilela, F.E.S.P., Arzolla, F.A.R.D.P., Franco, G.A.D.C., Cordeiro, I., Koch, I., Baitello, J.B., Lombardi, J.A., Lima, L.R., Lohmann, L.G., Bernacci, L.C., Assis, M. A., Aidar, M.P.M., Wanderley, M.G.L., Toniato, M.T.Z., Ribeiro, M., Groppo, M., Cavassan, O.,
Sano, P.T., Rodrigues, R.R., Fisch, S.T.V. \& Martins, S.E. 2008. Fanerógamas. In: R.R. Rodrigues, C.A. Joly, M.C.W. Brito, A. Paese, J.P. Metzger, L. Casatti, M.A. Nalon, N. Menezes, N.M. Ivanauskas, V. Bolzani, \& V. L.R. Bononi (coords.). Diretrizes para a conservação e restauração da biodiversidade no Estado de São Paulo. Secretaria do Meio Ambiente; Instituto de Botânica; Fapesp, São Paulo, pp. 104-109.

Eisenlohr, P.V. Melo, M.M.R.F.; Nascimento, M.T.; Santos, A.C.L.; Rodrigues, P.J.P. 2015. Estrutura de comunidades vegetais em áreas suscetíveis a efeitos de borda: conceitos, métodos e estudos de caso. In: P. Eisenlohr, J. Felfili, M. Fiuza de Melo, L. Andrade \& João Meira-Neto. (eds.). Fitossociologia no Brasil: Métodos e Estudos de Casos Volume II. Editora UFV, Viçosa, pp. 119-138.

Esser, H. 1999. Taxonomic notes on Neotropical Maprounea Aublet (Euphorbiaceae). Novon 9: 32-35.

Fiaschi, P \& Pirani, J.R. 2007. Estudo taxonômico do gênero Schefflera J.R. Forest \& G. Forest. (Araliaceae) na região sudeste do Brasil. Boletim de Botânica da Universidade de São Paulo 25: 95-142.

Fiaschi, P., Jung-Mendaçolli, S.L., Cabral, L.P. \& Frodin, D.G. 2007. Araliaceae. In: M.G. Wanderley, G.J. Shepherd, T.S. Melhem \& A.M. Giulietti (coords). Flora Fanerogâmica do Estado de São Paulo. Instituto de Botânica, São Paulo, v. 5, pp. 1-16.

Filardi, F.L.R., Felsemburgh, C.A. \& Garcia, F.C.P. 2016. Enterolobium Mart. In: M.G.L. Wanderley, G.J. Shepherd, T.S. Melhem, A.M. Giulietti \& S.E. Martins (coords.). Flora Fanerogâmica do Estado de São Paulo. Secretaria do Meio Ambiente; Instituto de Botânica; FAPESP, São Paulo, v. 8, pp. 100-103.

Flora do Brasil 2020 em construção. 2020. Jardim Botânico do Rio de Janeiro. Disponível em <http:// floradobrasil.jbrj.gov.br/reflora/floradobrasil/ (acesso em 17-II-2020).

Frigieri, F.F., Iwanicki, N.S., Gandara, F.B., Ferraz, E.M., Romão, G.O., Coletti, G.F., Souza, V.C. \& Moreno, M.A. 2016. Guia de plântulas e sementes da Mata Atlântica do Estado de São Paulo. 1 ed. IPEF, Piracicaba.

Gaglioti, A.L. \& Romaniuc Neto, S. 2012. Urticaceae. In: M.G.L. Wanderley, G.J. Shepherd, T.S. Melhem, A.M. Giulietti \& S.E. Martins (coords.). Flora Fanerogâmica do Estado de São Paulo. Secretaria do Meio Ambiente; Instituto de Botânica; FAPESP, São Paulo, v. 7, pp. 331-361.

Garcia, C.C., Reis, M.G.F., Reis, G.G., Pezzopane, J.E.M., Lopes, H.N.S. \& Ramos, D.C. 2011. Regeneração natural de espécies arbóreas em fragmento de floresta estacional semidecidual montana, no domínio da Mata Atlântica, em Viçosa, MG. Ciência Florestal 21: 677-688.

Guidotti, V., Ferraz, S.F.B., Pinto, L.F.G., Sparovek, G., Taniwaki, R.H., Garcia, L.G. \& Brancalion, P.H.S. 2020. Changes in Brazil's forest code can erode the potencial of riparian buffers to supply watershed services. Land Use Policy 94: 104511. 
Haddad, T.M., Hertel, M.F., Bianchini, E. \& Pimenta, J.A. 2016. Architecture of four tree species from different strata of a semideciduous forest in southern Brazil. Australian Journal of Botany 64: 89-99.

Higuchi, P. 2003. Dinâmica da regeneração natural da vegetação arbórea em um fragmento de Floresta Estacional Semidecidual Montana secundária, em Viçosa, MG. Dissertação de Mestrado, Universidade Federal de Viçosa, Viçosa.

Higuchi, P., Reis, M.G.F., Reis, G.G., Pinheiro, A.L., Silva, C.T. \& Oliveira, C.H.R. 2006. Composição florística da regeneração natural de espécies arbóreas ao longo de oito anos em um fragmento de Floresta Estacional Semidecidual, em Viçosa, MG. Revista Árvore 30: 893-904.

Hora, R. \& Soares, J.J. 2002. Estrutura fitossociológica da comunidade de lianas em uma floresta estacional semidecidual na Fazenda Canchim, São Carlos, SP. Revista Brasileira de Botânica 25: 323-329.

IBGE (Instituto Brasileiro de Geografia e Estatística). 2012. Manual Técnico da Vegetação Brasileira: número 1, 2 ed., Rio de Janeiro.

Kronka, F.J.N., Nalon, M.A. \& Matsukuma, C.K. 2005. Inventário florestal da vegetação natural do Estado de São Paulo. Secretaria do Meio Ambiente, Instituto Florestal, Imprensa Oficial, São Paulo.

Leyser, G., Zanin, E.M., Budke, J.C., Mélo, M.A. \& Henke-Oliveira, C. 2012. Regeneração de espécies arbóreas e relações com componente adulto em uma floresta estacional no vale do rio Uruguai, Brasil. Acta Botanica Brasilica 26: 74-83.

Lorenzi, H. 1992. Árvores brasileiras: manual de identificação e cultivo de plantas arbóreas nativas do Brasil. v. 1. Instituto Plantarum, Nova Odessa.

Lorenzi, H. 1998. Árvores brasileiras: manual de identificação e cultivo de plantas arbóreas nativas do Brasil. v. 2., Instituto Plantarum, Nova Odessa.

Lorenzi, H. 2009. Árvores brasileiras: manual de identificação e cultivo de plantas arbóreas nativas do Brasil. v. 3. Instituto Plantarum, Nova Odessa.

Lorenzi, H., Souza, H.M., Torres, M.A.V. \& Bacher, L.B. 2009. Árvores exóticas no Brasil: madeiras, ornamentais e aromáticas. Instituto Plantarum, Nova Odessa.

Martins, S.V., Gleriani, J.M., Amaral, C.H. \& Ribeiro, T.M. 2008. Caracterização do dossel e do estrato de regeneração natural no sub-bosque e em clareiras de uma florestal estacional semidecidual no município de Viçosa, MG. Revista Árvore 32: 759-767.

Matos, C., Amorim, D.A., Medina, E.S., Silva, J.F.S. \& Lopes, J.S. 2018. Lista orientativa de espécies arbóreas e arbustivas de ocorrência regional, calendário de florescimento e recursos para a melissofauna para projetos de restauração ecológica e enriquecimento de pastos melíferos. Secretaria de Infraestrutura e Meio Ambiente. Disponível em https://www.infraestruturameioambiente. sp.gov.br/2018/10/divulgada-lista-de-orientacao-deprojetos-de-restauracao-ecologica-que-beneficiara-asabelhas/ (acesso em 16-XII-2018).
Mendonça-Souza, L.R. 2006. Ficus (Moraceae) no Estado de São Paulo. Dissertação de Mestrado, Instituto de Botânica, São Paulo.

Moreira, B. \& Carvalho, F.A. 2013. A comunidade arbórea de um fragmento urbano de Floresta Atlântica após 40 anos de sucessão secundária (Juiz de Fora, Minas Gerais). Biotemas 26: 59-70.

Myers, N., Mittermeier, R.A., Mittermeier, C.G., Fonseca, G.A.B. \& Kent, J. 2000. Biodiversity hotspots for conservation priorities. Nature 403: 852-858.

Nalon, M.A., Mattos, I.F.A. \& Franco, G.A.D.C. 2008. Meio físico e aspectos da fragmentação da vegetação. In: R.R. Rodrigues, C.A. Joly, M.C.W. Brito, A. Paese, J.P. Metzger, L. Casatti, M. A. Nalon, N. Menezes, N.M. Ivanauskas, V. Bolzani \& V.L.R. Bononi (coords.). Diretrizes para a conservação e restauração da biodiversidade no Estado de São Paulo. Secretaria do Meio Ambiente, Instituto de Botânica, Fapesp, São Paulo. pp. 15-21.

Naves, R.P. 2013. Estrutura do componente arbóreo e da regeneração de áreas em processo de restauração com diferentes idades, comparadas a ecossistema de referência. Dissertação de Mestrado, Universidade de São Paulo, Piracicaba.

Nóbrega, A.M.F., Valeri, S.V., Paula, R.C., Silva, S.A. \& Rêgo, N.H. 2007. Uso da fitossociologia na avaliação da efetividade da restauração florestal em uma várzea degradada do rio Mogi Guaçu, SP. Scientia Forestalis 75: 51-63.

Oliveira, A.P.C. 2012. Flora vascular não-arbórea do Parque Estadual de Porto Ferreira, SP, Brasil. Dissertação de Mestrado, UNESP, Rio Claro.

Queiroz, I.H.B., Mendes, L.F., Sebastiani, R. \& Sais, A.C. 2017. A Fazenda da Aeronáutica de Pirassununga como potencial área de conservação da biodiversidade na Bacia Hidrográfica do Rio Mogi Guaçu, SP. Holos Environment (Suplemento Simpósio de Biologia Vegetal 2017): 203-214.

Rodrigues, V.H.P., Lopes, S.F., Araújo, G.M. \& Schiavini, I. 2010. Composição, estrutura e aspectos ecológicos da floresta ciliar do rio Araguari no Triângulo Mineiro. Hoehnea 37: 87-105.

Romaniuc Neto, S., Godoi, J.V., Villagra, B.L.P., Almeida-Scabbia, R.J. \& Melo, M.M.R.F. 2012. Caracterização florística, fitossociológica e fenológica de trepadeiras de mata ciliar da Fazenda Campininha, Mogi Guaçu, SP, Brasil. Hoehnea 39: 145-155.

Salles, J.C. \& Schiavini, I. 2007. Estrutura e composição do estrato de regeneração em um fragmento florestal urbano: implicações para a dinâmica e a conservação da comunidade arbórea. Acta Botanica Brasilica 21: 223-233.

Santos, S.C., Budke, J.C. \& Muller, A. 2012. Regeneração de espécies arbóreas sob a influência de Merostachys multiramea Hack. (Poaceae) em uma floresta subtropical. Acta Botanica Brasilica 26: 218-229.

Sartori, R.A. 2010. Florística, estrutura e guildas da comunidade arbóreo-arbustiva de um fragmento de mata-atlântica no município de Socorro-SP. Dissertação de Mestrado, Universidade Federal de Lavras, Lavras. 
Sartori, R.A., Carvalho, D.A. \& Berg, E.V.D. 2007. Caracterização florística e fitossociológica do estrato arbóreo de um fragmento de Mata Atlântica, no município de Socorro, SP. Revista Brasileira de Biociências 5 : 897-899.

Silva Junior, W.M. 2002. Caracterização florística e fitossociológica da regeneração natural em dois trechos de uma Floresta Estacional Semidecidual no município de Viçosa, MG. Dissertação de Mestrado, Universidade Federal de Viçosa, Viçosa.

Silva, L.A. \& Soares, J.J. 2002. Levantamento fotossociológico em fragmentos de Floresta Estacional Semidecidual no município de São Carlos, SP. Acta Botânica Brasilica 16: 205-216.

Silva, L.A. \& Soares, J.J. 2003. Composição florística de um fragmento de Floresta Estacional Semidecídua no município de São Carlos-SP. Revista Árvore 27: 647-656.

SpeciesLink. 2020. Herbário da Escola Superior de Agricultura Luiz de Queiroz (ESA). Disponível em: http://www.splink.org.br (acesso em 28-IV-2020).

Souza Junior, C.N. \& Brancalion, P.H.S. 2016. Sementes e mudas. Guia para propagação de árvores brasileiras. Oficina de Textos, São Paulo.

Tamashiro, J.Y. \& Escobar, N.A.G. 2016. Tribo Mimoseae Bronn. In: M.G.L. Wanderley, G.J. Shepherd, T.S. Melhem, A.M. Giulietti \& S.E. Martins (cords.). Flora Fanerogâmica do Estado de São Paulo. Secretaria do Meio Ambiente, Instituto de Botânica, FAPESP, São Paulo, v. 8, pp. 119-166.

Ter Steege, H., Pitman, N.C., Sabatier, D., Baraloto, C., Salomão, R.P., Guevara, J.E., Phillips, O.L., Castilho, C.V., Magnusson, W.E. Molino, J.F. et al. 2013. Hyperdominance in the Amazonian tree flora. Science, 342 (6156): 1243092.

Tibiriçá, Y J.A., Coelho, L.F.M. \& Moura, L.C. 2006. Florística de lianas em um fragmento de floresta estacional semidecidual, Parque Estadual de Vassununga, Santa Rita do Passa Quatro, SP, Brasil. Acta Botanica Brasilica 20: 339-346.

Toledo Filho, D.V., Faria, I.M.P., Freitas, J.A. \& Zanella, J. 2010. Estudo de três fragmentos florestais de altitude no município de Itapira-SP. Fórum Ambiental da Alta Paulista 6: 243- 256.

Udulutsch, R.G., Assis, M.A. \& Picchi, D.G. 2004. Florística de trepadeiras numa floresta estacional semidecídua, Rio Claro - Araras, Estado de São Paulo, Brasil. Revista Árvore 27: 125-134.

Viani, R.A.G. 2007. Juvenile trees from a riparian forest in Bofete-SP, southeastern Brazil. Field Museum Field Guides. Disponível em https://fieldguides.fieldmuseum. org guides? region $=915 \&$ country $=924 \&$ category $=37 \&$ subcategory $=91 \&$ items_per_page $=1600 \&=$ Filter $($ acesso em 18-XI- 2018).

Viani, R.A.G. \& Rodrigues, R.R. 2007. Sobrevivência em viveiro de mudas de espécies nativas retiradas da regeneração natural em remanescente florestal. Pesquisa Agropecuária Brasileira 42(8): 1067-1075.

Viani, R.A.G. \& Rodrigues, R.R. 2009. Potential of the seedling community of a forest fragment for tropical forest restoration. Scientia Agricola 66(6): 772-779.

Xavier, A.F., Bolzani, B.M. \& Jordão, S. 2008. Unidades de Conservação da Natureza no Estado de São Paulo In: R.R. Rodrigues, C.A. Joly, M.C.W. Brito, A. Paese, J.P. Metzger, L. Casatti, M. A. Nalon, N. Menezes, N.M. Ivanauskas, V. Bolzani, \& V.L.R. Bononi (coords.). Diretrizes para a conservação e restauração da biodiversidade no Estado de São Paulo. Secretaria do Meio Ambiente, Instituto de Botânica, Fapesp, São Paulo, pp. 23-42.

Recebido: 22.10 .2020 Aceito: 11.03 .2021 Editor Associado: Cláudia Baider 